\title{
The population dynamics of ecosystem engineers and habitat modification
}

\author{
S. Watt ${ }^{\mathrm{a}}\left(\right.$ ib) and Z. Jovanoski ${ }^{\mathrm{a}}$ (iD) and I. Towers ${ }^{\mathrm{a}}$ (i) and R. Saifuddin ${ }^{\mathrm{b}}$ and H. Sidhu ${ }^{\mathrm{a}}$ \\ ${ }^{a}$ School of Science, UNSW Canberra, PO Box 7916 Canberra BC, ACT 2610, Australia \\ ${ }^{\mathrm{b}}$ Department of Cyber Security and Games, Canberra Institute of Technology, 37 Constitution Ave, Reid ACT \\ 2601, Australia \\ Email: simon.watt@adfa.edu.au
}

\begin{abstract}
We consider a native species whose environment is stressed due to the presence of an ecosystem engineer, a specific type of invasive species that has the ability to convert native habitat into one which is more conducive to its own survival. The re-engineering of the native habitat results in an increase in the intra-species competition among the resident species. A key aspect of ecosystem engineer dynamics is that their invasion is limited by the size of the native habitat and their ability to transform it. It has long been recognised that invasive species play a role in declining biodiversity and the degradation of native habitat.

We explore the effect of a single engineer species on a resident species and its habitat. The invading engineer species does not prey on the resident species but does compete for available habitat. The engineer species converts, modifies or re-engineers native habitat. The rate at which this conversion is performed determines whether or not colonisation will be successful. Over time the converted habitat degrades forming a decayed habitat that is not suitable to either species. The decayed habitat returns to its native state through a process of recovery. Once in its native state the habitat can be occupied by either the resident species or modified again by the ecosystem engineer. This recycling of the habitat is an important and novel feature in the model.

We investigate the dynamics of this model. We assume the dynamics of both species is governed by a logistictype differential equation whose carrying capacity is equal to the size of its habitat. In the logistic equation, it is the carrying capacity that determines the population size. A reduction in habitat will drive the population to a smaller size, similarly an increase in habitat will increase the population size.

An analysis of the model reveals three different approaches that may be used to control the invading species. The first is based on reducing the ability for the engineer species to convert native habitat. The model shows the existence of a minimum conversion rate below which the engineer species can not colonise the native habitat. Therefore conservation strategies should focus on reducing the engineer's ability to convert habitat. A second strategy is a well known strategy, the harvesting of the invading species. And the third is based on 'quarantining' the decayed habitat for a certain period of time. Although quarantining does not alter the conversion rate it does however favour the resident species by not permitting the engineer species to become the dominant species. However, large quarantine periods cause oscillations within the system which may not be desirable.
\end{abstract}

Finally, we propose directions for future work.

Keywords: Population modelling, invasive species, mathematical modelling, harvesting 
S. Watt $e t$ al., The population dynamics of ecoengineers ...

\section{INTRODUCTION}

Invasive species are species that have spread beyond their natural range, usually with human assistance, and have the potential to affect the native ecosystem and its biodiversity. Invading species, in and of themselves, are rated high as a cause of native biodiversity loss and economic damage. Moreover, invading species interact with all other factors that compromise the integrity of native ecosystems, such as habitat destruction, pollution and climate change.

Invasive species have long been considered as one of the primary threats to the conservation and maintenance of native species and their habitat (Butchart et al., 2010; Duncan et al., 2013; Lohr et al., 2017). Invasive species can change species' interactions by altering the nutrient flow and the trophic structure of ecosystems.

Of particular interest are ecosystem engineers, an invasive species that modify a habitat (biotic or abiotic) through physical changes in the available resources (Jones et al., 1994). All species modify the environment to some extent (Franco and Fontanari 2016). However, there are some species which modify the environment to the detriment of other species. Two commonly cited examples are humans and beavers. Humans have the ability to utilise available resources to continually change the landscape by building cities and roads to connect them to accommodate increasing populations. This conversion of the natural environment is to the detriment of the natural world, endangering habitats and species. Beavers must build dams to protect themselves from predators such as coyotes, wolves and bears and to ensure easy access to food during winter. However, the creation of dams can lead to flooding and destruction of forests (Choi, 2008).

Different types of habitat modification is described in Jones et al. (1994), where the term 'extended phenotype engineering' was used to distinguish it from 'accidental engineering', where the habitat modification does not help the engineer (or invasive) species in their reproduction and survival.

We propose a mathematical model to explore ways that the the engineer species can be controlled so its impact on native habitat is minimised. Specifically we look at the relative importance of model parameters, such as the habitat conversion rate, the effect of harvesting the invasive species, and the role of 'quarantining' a habitat. A key aspect of these models is that the growth of the engineer species is limited by the rate at which a native habitat is modified by the engineering population.

Our starting point is a model first developed by Gurney and Lawton (1996) and later extended by Gonzalez et al. (2008) with the inclusion of indirect inter-species competition between the engineer species and the native species.

\section{MATHEMATICAL MODEL}

The model presented by Gurney and Lawton (1996) and augmented by Gonzalez et al. (2008) is depicted in Figure 1 . The engineer (invasive) species, $I$, has the ability to modify the native habitat, $H_{1}$, used by the

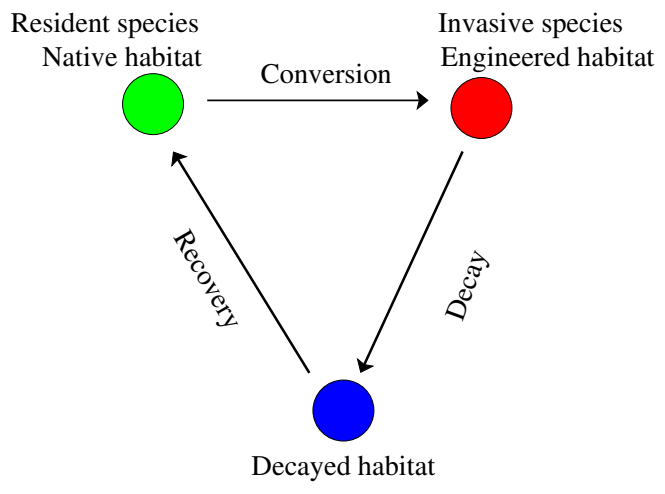

Figure 1: A schematic showing the dynamics among the habitats: the invasive species alters the native habitat, $H_{1}$, to the engineered habitat, $H_{2}$, then $H_{2}$ decays to an intermediate degraded state, $D$. Finally, the degraded state returns to its native state through the process of recovery. The resident species population, $R$, is limited by the size of the native habitat, and the invasive species, $I$, is limited by the size of the engineered habitat. 
resident species, $R$, into a modified habitat, $\mathrm{H}_{2}$, one more suited to meet its own ecological needs. The invasive species can transform the native habitat at a rate proportional to the population size of the invasive species, $f(I)=b I$ with $b$ being a constant conversion rate. The modified habitat then transitions to a decayed habitat at a rate $\delta$. The decayed habitat can not be utilised by either species. Finally, the decayed habitat returns to its native state through the process of recovery at a rate $\rho$. It is assumed that the population dynamics of both the native and invasive species are characterised by logistic-type growth models whose carrying capacity is equal to their respective habitat size, $H_{1}$ and $H_{2}$. The total habitat (native; modified; decayed) is assumed to be of a constant size, that is, $H_{1}(t)+H_{2}(t)+D(t)=T$ for all time, $t$. The mathematical model is

$\frac{d R}{d t}=r_{1} R\left(1-\frac{R}{H_{1}}\right), \quad \frac{d I}{d t}=r_{2} I\left(1-\frac{I}{H_{2}}\right)$,

$\frac{d H_{1}}{d t}=\rho D-b I H_{1}, \quad \frac{d H_{2}}{d t}=b I H_{1}-\delta H_{2}, \quad \frac{d D}{d t}=\delta H_{2}-\rho D$

where $r_{1}$ and $r_{2}$ are the intrinsic growth rates. In the rest of the paper, unless otherwise specified, we will use the values $r_{1}=1, r_{2}=2, \delta=0.5, \rho=2, b=1, R(0)=H_{1}(0)=9, I(0)=H_{2}(0)=1$ and $D(0)=0$, which gives $T=10$.

\section{SPECIAL CASES}

We discuss two cases, one where the engineered habitat does not degrade and another when the degraded habitat does not regenerate.

\subsection{Case 1 - No decay}

For the case where there is no decay, we set the decay rate $(\delta)$ to zero. Once the engineer species is introduced into a native habitat, the process of habitat conversion begins and eventually all of the native habitat is converted into engineered habitat. As a result, the native species will become extinct, in this non-spatial model. The conversion rate $(b)$ controls the rate of extinction, the higher the value, the quicker the process takes. This is shown in Figures 2 for $b=0.1$ and $b=1$. Note that in these figures the population and habitat have been normalised by the total habitat size, $T$.
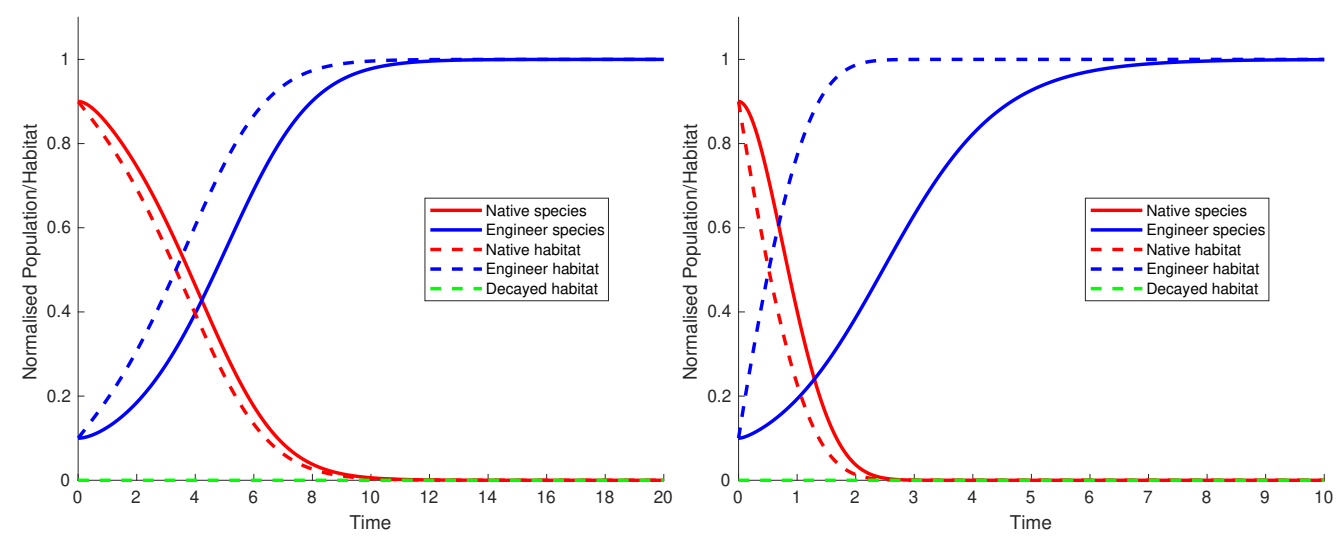

Figure 2: Population and habitat densities for the case with no decay for two different conversion rates $b=0.1$ (left) and $b=1$ (right).

\subsection{Case 2 - Decay with no recovery}

The introduction of an engineer species into a native habitat initiates the process of habitat conversion from habitat $H_{1}$ to habitat $H_{2}$. Case 2 depicts a situation where the engineered habitat decays with rate $\delta$, entering the degraded state $D$. As there is no recovery from the degraded habitat, both the native habitat and engineered habitat will eventually be depleted. Consequently, both species will become extinct. Figure 3 illustrates the extinction of both species and their habitats. 
S. Watt et al., The population dynamics of ecoengineers ...

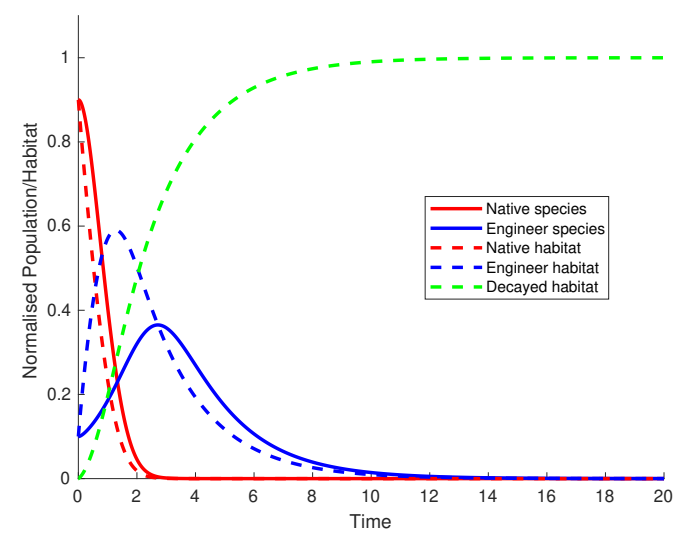

Figure 3: The case with no recovery from the decayed state, showing the extinction of both the native and engineer species.

\section{THE GENERAL MODEL}

In the case, the decayed habitat recovers to its native state at a rate $\rho$. In what follows we show that in this case co-existence between the native and engineer species is possible.

\subsection{Equilibrium solutions and successful colonisation}

The equilibrium solutions of the system described in equations (1)-(2) are

$R=H_{1}=\frac{\delta}{b}, \quad I=H_{2}=\frac{\rho(b T-\delta)}{b(\delta+\rho)}, \quad D=\frac{\delta(b T-\delta)}{b(\rho+\delta)}$.

To ensure that the population and habitat sizes are all non-negative requires that there be a minimum critical conversion rate, $b_{c r}=\delta / T$. For conversion rates above $b_{c r}$, there exists the possibility of co-existence between the resident and engineer species. For conversion rates below $b_{c r}$, the engineer species becomes extinct while the resident species reaches its maximum carrying capacity $R=H_{1}=T$. Figure 4 shows a bifurcation diagram of equilibrium states of the resident and invasive species as a function of the conversion rate $b$. We can calculate the dominance point, when the population size of the engineer species exceeds that of the resident species, identified by $b_{d o m}$ in Figure 4 . The dominance point can be calculated as

$b_{d o m}=b_{c r}\left(\frac{\delta+2 \rho}{\rho}\right)$.

\section{Controlling the POPUlation THROUGH HARVESTING}

One way to control the impact of the invasive engineer species is through culling or harvesting. To model this, a harvesting term can be added to the logistic-type growth equation for the engineer species. The new system becomes

$\frac{d R}{d t}=r_{1} R\left(1-\frac{R}{H_{1}}\right), \quad \frac{d I}{d t}=r_{2} I\left(1-\frac{I}{H_{2}}\right)-\beta I$

$\frac{d H_{1}}{d t}=\rho D-b I H_{1}, \quad \frac{d H_{2}}{d t}=b I H_{1}-\delta H_{2}, \quad \frac{d D}{d t}=\delta H_{2}-\rho D$

where $\beta$ is the harvesting rate. The equilibrium of the system is

$R=H_{1}=\frac{\delta}{b}+\frac{\beta \delta}{b \Delta}, \quad I=\frac{\rho(b T-\delta)}{b(\delta+\rho)}-\frac{\beta \rho T}{r_{2}(\delta+\rho)}, \quad H_{2}=\frac{\rho(b T-\delta)}{b(\delta+\rho)}-\frac{\beta \delta \rho}{b(\delta+\rho) \Delta}, \quad D=\frac{\delta(b T-\delta)}{b(\rho+\delta)}-\frac{\delta^{2} \beta}{b(\delta+\rho) \Delta}$, 
S. Watt et al., The population dynamics of ecoengineers ...

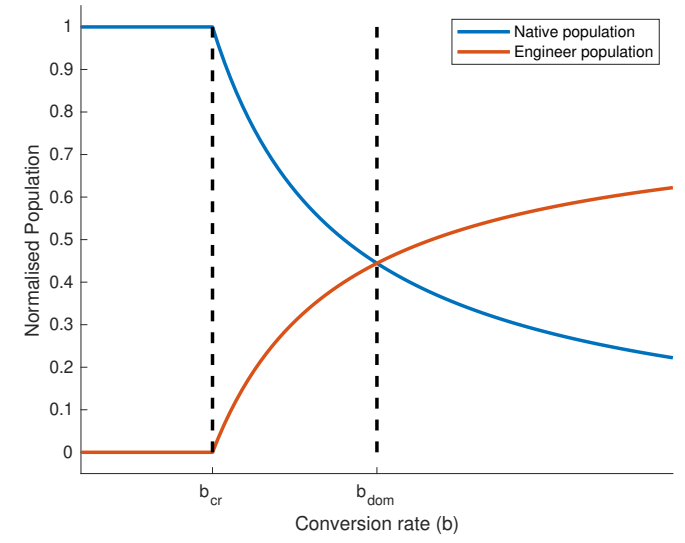

Figure 4: Bifurcation diagram for the equilibrium of the native $(R)$ and engineer species $(I)$ as a function of the conversion rate $(b)$.

with $\Delta=r_{2}-\beta>0$. When $\Delta \leq 0$ the harvesting rate equals or exceeds the intrinsic growth rate, the engineer species will become extinct. As before, to ensure non-negative population and habitat sizes the critical conversion rate now becomes

$b_{c r}=\frac{\delta}{T}\left(1+\frac{\beta}{\Delta}\right)$.

The dominance point now becomes

$b_{d o m}=\frac{b_{c r}}{\rho}\left(\delta+2 \rho+(\delta+\rho) \frac{\beta}{\Delta}\right)$.

By setting $\beta=0$, we get $\Delta=r_{2}$ and recover the model without harvesting.

From the analysis above, if $\Delta$ decreases (due to an increase in $\beta$ ) the size of the engineer species can be controlled. This is achieved by increasing the point of dominance, thus ensuring that the resident species remains more abundant.

\section{MODEL WITH FIXED RECOVERY TIME}

We consider an idea explored by Gurney and Lawton (1996), where the degraded habitat remains in that state for a fixed amount of time, $\tau$. Mathematically, this can be expressed as

$D(t)=\delta \int_{t-\tau}^{t} H_{2}(s) d s$,

The model can now be written as

$\frac{d R}{d t}=r_{1} R\left(1-\frac{R}{H_{1}}\right), \quad \frac{d I}{d t}=r_{2} I\left(1-\frac{I}{H_{2}}\right)$,

$\frac{d H_{1}}{d t}=\delta H_{2}(t-\tau)-b I H_{1}, \quad \frac{d H_{2}}{d t}=b I H_{1}-\delta H_{2}, \quad \frac{d D}{d t}=\delta\left[H_{2}(t)-H_{2}(t-\tau)\right]$.

The equilibrium solution becomes

$R^{*}=H_{1}^{*}=\frac{\delta}{b}, \quad I^{*}=H_{2}^{*}=\frac{(b T-\delta)}{b(1+\delta \tau)}, \quad D^{*}=\frac{\delta \tau(b T-\delta)}{b(1+\delta \tau)}$.

For non-negative population and habitat sizes, we require that $b_{c r}=\delta / T$ (as for the general model) and the dominance point becomes $b_{d o m}=\delta(2+\delta \tau) / T$. Before analysing this system, we will make some simplifications. Note that the resident population $(R)$ does not have an effect on habitat dynamics nor on the 
S. Watt et al., The population dynamics of ecoengineers ...

engineer species. Furthermore, since the total habitat size is constant we can write $H_{1}(t)=T-H_{2}(t)-D(t)$, hence eliminate the equation for the native habitat. The new system becomes

$$
\frac{d I}{d t}=r_{2} I\left(1-\frac{I}{H_{2}}\right), \quad \frac{d H_{2}}{d t}=b I\left(T-H_{2}-D\right)-\delta H_{2}, \quad \frac{d D}{d t}=\delta\left[H_{2}(t)-H_{2}(t-\tau)\right] .
$$

The stability analysis of this reduced system gives rise to a characteristic polynomial of degree three whose coefficients depend on $\tau$. A general geometric criteria for stability switching as a function of $\tau$ is discussed in Beretta and Kuang (2002) and Jianquan and Zhien (2004).

The characteristic polynomial of this delayed system can be shown to be

$\lambda^{3}+a_{1} \lambda^{2}+a_{2} \lambda+a_{3} e^{-\lambda \tau}+a_{4} \lambda e^{-\lambda \tau}+a_{5}=0$,

where $a_{1}=b H_{2}^{*}+\delta+r_{2}, a_{2}=\left(2 b r_{2}+b \delta+b \delta r_{2} \tau\right) H_{2}^{*}+r_{2}(\delta-b T), a_{3}=-b \delta r_{2} H_{2}^{*}, a_{4}=-b \delta H_{2}^{*}$ and $a_{5}=b \delta r_{2} H_{2}^{*}$. A Hopf bifurcation occurs when the eigenvalue $\lambda$ is purely imaginary, that is $\lambda=i \omega$, giving rise to periodic solutions.

We found that a Hopf bifurcation occurs for a critical recovery time $\tau_{c} \approx 11.22$. For $\tau<\tau_{c}$, the oscillatory solutions decay to a steady-state solution, and for $\tau>\tau_{c}$, the oscillatory solutions persist. These results are shown in Figures 5(a) and 5(b). By including harvesting of the engineer species into the above system, it can be seen in Figure $5(\mathrm{c})$ that harvesting decreases the amplitude of the oscillations. The system decays to a stead-state solution as the harvesting is further increased, as shown in Figure 5'd).

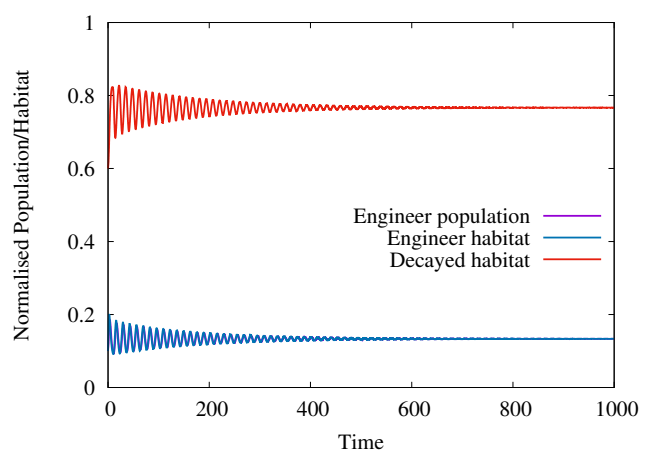

(a)

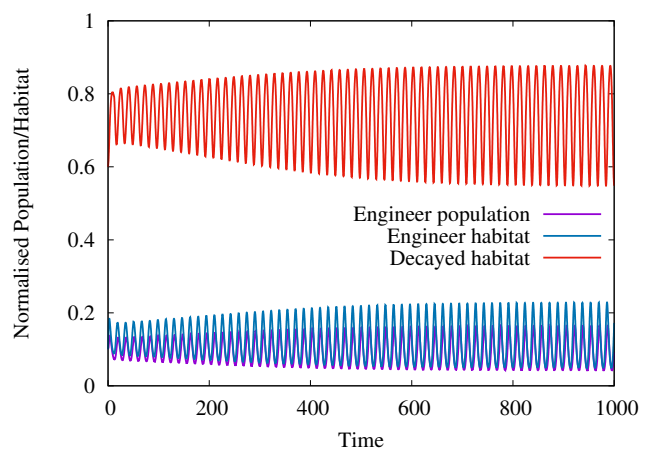

(c)

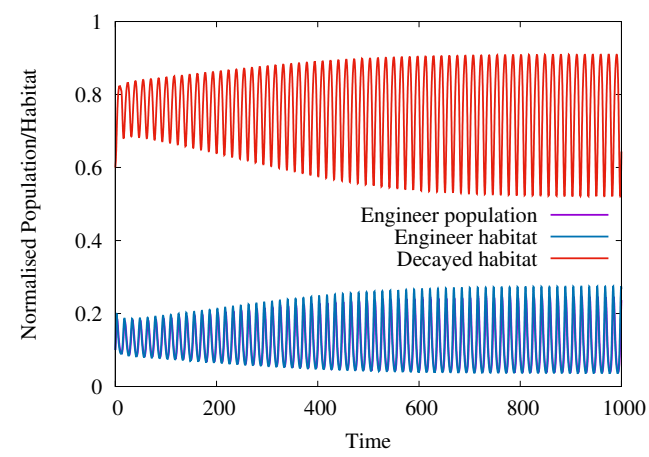

(b)

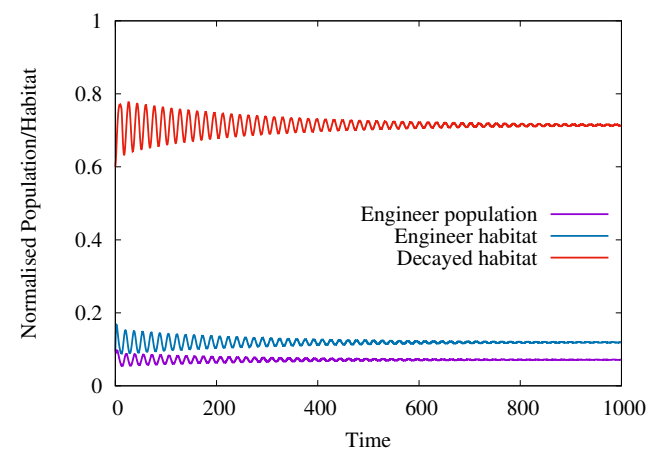

(d)

Figure 5: The solution with a delay in the system with no harvesting (a) $\tau=10$ and (b) $\tau=12$ and the system with harvesting (c) $\tau=12$ and $\beta=0.2$ and (d) $\tau=12$ and $\beta=0.4$.

\section{DISCUSSION AND CONCLUSION}

We have investigated the effects of introducing an invasive species, specifically an ecosystem engineer, into a native habitat with a single resident species. To survive, the invasive species must convert the native habitat into one that sustains it. 
Successful colonisation by the engineer species depends on the rate of native habitat modification. If the ecosystem engineer is inefficient in its effort to convert habitat then its ability to secure a permanent presence within the native habitat is greatly diminished. There exists a threshold, $b_{c r}$, below which invasion into the native habitat is not sustainable. It was demonstrated that stable co-existence can be achieved. A bifurcation analysis indicates that if the conversion rate is above $b_{d o m}$ the invasive species dominates the resident species. To avoid the possible extinction of the resident species, conservationists should try to reduce the conversion rate $b$, thus making the invasive species less effectual in their ability to modify native habitat. Whether this is done by bio-manipulation or the release of certain toxins, not harmful to the resident species, will depend on the type of traits of the invasive species concerned. Another way to reduce $b$ (and ideally below $b_{c r}$ ) is to use a barrier, such as a fence.

The effect of harvesting of the engineer species was also considered. It was seen that without inhibition and with a large enough engineering conversion rate, the invasive species will dominate the native species. Harvesting can be an effective means of intervention to prevent the engineering species from becoming dominant. Here the important parameter is $\Delta=r_{2}-\beta$. It can be interpreted as the 'effective' intrinsic growth rate. A reduction in $\Delta$ results to an increase in both $b_{c r}$ and $b_{d o m}$. Even if it is not possible to eliminate the invasive species a higher $b_{d o m}$ will ensure the native population remains more abundant.

We also investigated the scenario where the degraded habitat remained in that state for a fixed amount of time, $\tau$, before it recovers. This may be thought of as a type of 'quarantine' measure. The model with fixed recovery time exhibits a Hopf bifurcation. When the recovery time is greater than some critical value $\tau_{c}$, the resident population undergoes large amplitude oscillations of a fixed period. The critical conversion rate, $b_{c r}$ is not affected by $\tau$, but the point of dominance, $b_{d o m}=\delta(2+\delta \tau) / T$, is. By quarantining the decayed habitat for longer lengths of time the value of $b_{d o m}$ can be increased, thus maintaining a higher abundance of the native species. Care, however, must be taken as increasing $\tau$ induces larger amplitude oscillations in both species which may not be desirable, thus determining an optimal $\tau$ is a worthy future goal.

Finally, random environmental fluctuations affect both intra-species and inter-species competition for limited resources. Models with fixed parameters may not be adequate for conservation purposes. Future model refinements could include stochastic processes that better capture the impact of environmental change on species survival.

\section{REFERENCES}

Beretta, E. and Y. Kuang (2002). Geometric stability switch criteria in delay differential systems with delay dependent parameters. SIAM Journal on Mathematical Analysis 33(5), 1144-1165.

Butchart, S. H. M., M. Walpole, B. Collen, A. van Strien, J. P. W. Scharlemann, R. E. A. Almond, J. E. M. Baillie, B. Bomhard, C. Brown, J. Bruno, K. E. Carpenter, G. M. Carr, J. Chanson, A. M. Chenery, J. Csirke, N. C. Davidson, F. Dentener, M. Foster, A. Galli, J. N. Galloway, P. Genovesi, R. D. Gregory, M. Hockings, V. Kapos, J.-F. Lamarque, F. Leverington, J. Loh, M. A. McGeoch, L. McRae, A. Minasyan, M. H. Morcillo, T. E. E. Oldfield, D. Pauly, S. Quader, C. Revenga, J. R. Sauer, B. Skolnik, D. Spear, D. Stanwell-Smith, S. N. Stuart, A. Symes, M. Tierney, T. D. Tyrrell, J.-C. Vié, and R. Watson (2010). Global biodiversity: Indicators of recent declines. Science 328(5982), 1164-1168.

Choi, C. (2008). Tierra del fuego: The beavers must die. Nature 453, 968.

Duncan, R. P., A. G. Boyer, and T. M. Blackburn (2013). Magnitude and variation of prehistoric bird extinctions in the pacific. Proceedings of the National Academy of Sciences, 201216511.

Franco, C. and J. F. Fontanari (2016). The spatial dynamics of ecosystem engineers. arXiv preprint arXiv:1611.09283.

Gonzalez, A., A. Lambert, and A. Ricciardi (2008). When does ecosystem engineering cause invasion and species replacement? Oikos 117(8), 1247-1257.

Gurney, W. and J. Lawton (1996). The population dynamics of ecosystem engineers. Oikos 76, 273-283.

Jianquan, L. and M. Zhien (2004). Stability switches in a class of characteristic equations with delay-dependent parameters. Nonlinear Analysis: Real World Applications 5(3), 389-408.

Jones, C. G., J. H. Lawton, and M. Shachak (1994). Organisms as ecosystem engineers. In Ecosystem Management, pp. 130-147. Springer.

Lohr, C. A., J. Hone, M. Bode, C. R. Dickman, A. Wenger, and R. L. Pressey (2017). Modeling dynamics of native and invasive species to guide prioritization of management actions. Ecosphere 8(5). 